\title{
High-speed planar thermometry and velocimetry using thermographic phosphor particles
}

\author{
Christopher Abram • Benoit Fond • \\ Andrew L. Heyes $\cdot$ Frank Beyrau
}

Received: 12 February 2013/Accepted: 11 March 2013/Published online: 29 March 2013

(C) The Author(s) 2013. This article is published with open access at Springerlink.com

\begin{abstract}
Simultaneous gas-phase temperature and velocity imaging using micrometer-size thermographic phosphor particles seeded into the flow is demonstrated at a $3 \mathrm{kHz}$ repetition rate. The velocity field is measured using a standard particle image velocimetry approach, while the temperature is determined from the temperature sensitive phosphorescence emission of the particles following excitation at $355 \mathrm{~nm}$. Since the particles are very small, they rapidly assume the temperature and velocity of the surrounding gas. A single shot temperature precision of better than $5 \%$ was achieved at $500 \mathrm{~K}$. Time-resolved measurements in the wake of a heated cylinder are presented, demonstrating the utility of these imaging diagnostics to observe transient, coupled heat and mass transfer phenomena.
\end{abstract}

\section{Introduction}

Optical measurement techniques have greatly improved the understanding of fluid flows and their coupling with heat transfer and chemical reactions. The investigation of such flows and these interactions requires diagnostics that allow two-dimensional combined vector-scalar measurements. Such imaging techniques offer simultaneous statistical sampling of important quantities and their gradients, which can be compared to predictive numerical models $[1,2]$.

Electronic supplementary material The online version of this article (doi:10.1007/s00340-013-5411-8) contains supplementary material, which is available to authorized users.

C. Abram · B. Fond · A. L. Heyes · F. Beyrau ( ()

Department of Mechanical Engineering, Imperial College

London, Exhibition Road, London SW7 2AZ, UK

e-mail: f.beyrau@imperial.ac.uk
However, at low sampling rates of several Hertz the measured fields are, for most practical flows, not correlated in time and therefore do not provide any information on unsteady flow regimes.

Sampling at much higher frequencies of several $\mathrm{kHz}$ allows the tracking of transient behavior or specific events in systems involving the short timescales associated with turbulent flows. The recent development of both diodepumped solid-state (DPSS) lasers and high framing rate CMOS cameras has extended the application of some diagnostic techniques to multi-kHz rate measurements $[3$, 4]. CMOS cameras are now available with large onboard memory, so sustained measurements that record many thousands of frames can cover a wide range of temporal scales This allows rapid accumulation of measurements to capture rare events such as engine misfire [5], and also the post-triggering of a recording sequence to capture the flow behavior preceding transient combustion events such as flame extinction [6].

Planar velocity measurements have been successfully performed at $\mathrm{kHz}$ repetition rates in a number of applications using particle image velocimetry (PIV) (see reviews $[3,4]$ and references therein), where micrometer-size particles or droplets are seeded into the flow and their movement is tracked in time to determine the velocity field. However, for temperature, a variable of critical interest in flows involving heat transfer or chemical reactions, to date there are only two demonstrations of planar measurements at sustained $\mathrm{kHz}$ repetition rates $[7,8]$. Both are based on laser-induced fluorescence (LIF) of toluene seeded into the flow and a two-color detection scheme which uses the red shift of the fluorescence spectrum with increasing temperature. This technique was first applied in a heated nitrogen jet [7] and later, in combination with PIV, to investigate the evolution of temperature stratification in a 
motored internal combustion engine fed with nitrogen [8]. However, strong oxygen quenching prohibited measurements in air and the toluene emission around $300 \mathrm{~nm}$ required the use of high-speed intensifiers, which are associated with non-linearities and charge depletion effects $[4,9]$.

In this study, we present an alternative high-speed measurement technique based on thermographic phosphor particles. These particles are seeded into the gas flow and used as a tracer for simultaneous imaging of temperature and velocity at $3 \mathrm{kHz}$. Velocimetry is performed with a conventional PIV approach, using the Mie scattered light from the phosphor particles. Following simultaneous UV excitation, these particles emit phosphorescence with a temperature-dependent emission spectrum. When two spectrally filtered images of the particle phosphorescence emission are recorded, the tracer temperature can be determined using a ratio-based method. At low repetition rates, this technique has previously been demonstrated for time-averaged [10] and single shot [11] simultaneous temperature and velocity measurements.

For flow measurements based on solid tracer particles, the particles must rapidly assume the gas temperature and velocity. It has been shown by many groups that micrometer-size ceramic particles are suitable tracers for PIV for a wide range of turbulent flows, in that they follow fluctuations in flow velocity with very good accuracy. Since the slip velocity is very low, conduction is the dominant mode of heat transfer between the gas and the particles. Based on a detailed heat transfer model it was previously shown that $2 \mu \mathrm{m}$ phosphor particles can also accurately trace the gas temperature for most technically relevant flows [11].

This technique has several striking features. The tracer material is chemically inert and able to survive in reactive, high temperature environments [12, 13], and for many phosphors the emission is insensitive to pressure and oxygen quenching. In addition, many thermographic phosphors have relatively broad excitation spectra allowing direct excitation with solid-state lasers, and their subsequent emission is often in the visible range with a high quantum yield, permitting the use of non-intensified cameras. The general properties of thermographic phosphors and their applications can be found in two excellent reviews $[12,13]$.

In the following, we describe the experimental setup for combined high-speed thermometry and velocimetry. The precision and accuracy of the $\mathrm{kHz}$ thermometry technique is evaluated, involving the investigation of CMOS camera performance for quantitative measurements in terms of both linearity and frame-to-frame variations, as well as actual gas-phase measurements in a heated jet of air for direct assessment of the single shot pixel-to-pixel precision. Finally, the potential of these combined diagnostics is demonstrated on an unsteady heat transfer problem consisting of a heated cylinder in a cross flow.

\section{Experimental setup}

\subsection{Test cases}

Measurements were performed in an electrically heated jet of air ( $21 \mathrm{~mm}$ diameter, velocity of $7 \mathrm{~m} / \mathrm{s})$ surrounded by a room temperature coflow $(80 \mathrm{~mm}$ diameter, velocity of $0.5 \mathrm{~m} / \mathrm{s}$ ). Both gas streams were seeded with $2 \mu \mathrm{m} \mathrm{BaM-}$ $\mathrm{gAl}_{10} \mathrm{O}_{17}: \mathrm{Eu}^{2+}$ (BAM:Eu) phosphor particles using inhouse built reverse cyclone seeders. In the second heat transfer study, the central nozzle was removed and a pipe of $6.25 \mathrm{~mm}$ diameter heated to $530 \mathrm{~K}$ was positioned horizontally above the seeded coflow as shown in Fig. 1. Here, the free stream velocity was set to $1.6 \mathrm{~m} / \mathrm{s}$ so at a temperature of $293 \mathrm{~K}$ the Reynolds number was around 700 , resulting in irregular vortex shedding in the wake of the cylinder [14].

\subsection{Thermometry}

To excite the phosphor, the third harmonic output of a diode-pumped Nd:YAG laser (JDSU, Q301) with a pulse duration of $25 \mathrm{~ns}$ and a pulse energy of $1.3 \mathrm{~mJ}$ at $3 \mathrm{kHz}$ was formed into a $600 \mu \mathrm{m}$ thick and $50 \mathrm{~mm}$ high light sheet using a Galilean telescope and $f=-50 \mathrm{~mm}$ and $f=500 \mathrm{~mm}$ cylindrical lenses. The phosphorescence emission was detected by two non-intensified CMOS cameras (Photron SA5, 12 bit, 1,024 × 1,024 pixels), fitted with $50 \mathrm{~mm} f / 1.4$ Nikon lenses. The cameras were operated at $3 \mathrm{kHz}(2,800$ stored frames per $0.9 \mathrm{~s}$ recording) with an exposure time of $9 \mu$ s. However, the actual measurement duration is determined by the phosphorescence decay time, which is for this phosphor around $1 \mu$ s at room temperature and decreases with increasing temperature. A long pass

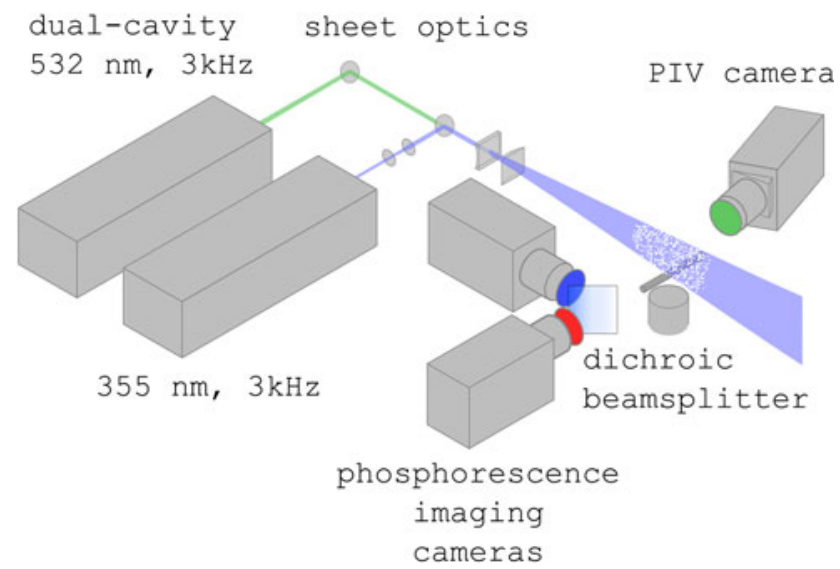

Fig. 1 Schematic of the experimental setup 


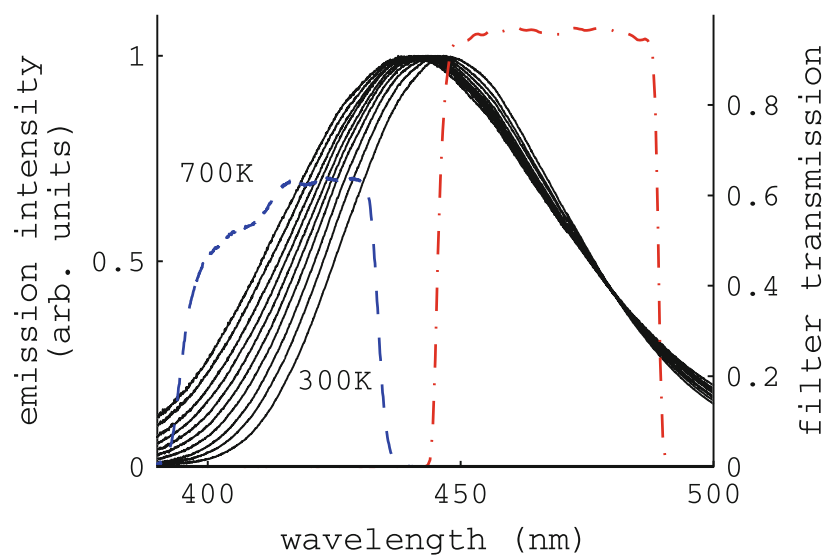

Fig. 2 Normalized BAM:Eu emission spectra plotted at $50 \mathrm{~K}$ temperature intervals. The transmission curves of the two detection channels (blue and red) are also displayed

dichroic $45^{\circ}$ beamsplitter (Chroma T445LP) in combination with two $0^{\circ}$ interference filters (Edmund Optics 466-40 $\mathrm{nm}$ and Chroma 420-30 nm) were used to separate and filter the two detection channels, as shown in Fig. 2. The camera/beamsplitter system was carefully adjusted to minimize relative distortion and differences in light collection path. After acquisition, background images were recorded and subtracted from the image pairs. Images were then spatially overlapped using software-based mapping (LaVision, DaVis 7.2) based on images of a calibration target. A cutoff filter at 15 counts was applied (corresponding to three times the readout noise of the camera), followed by $4 \times 4$ digital binning and a $3 \times 3$ unweighted moving average filter. This also ensures that there are enough particles in each superpixel to generate a sufficient phosphorescence signal for thermometry. Using the seeders described above, the seeding density was adjusted to be slightly higher than that required for PIV, without compromising the contrast in the high resolution PIV raw images. This seeding density is also used in other approaches where the step in particle number density at the reaction zone is used to identify the flame front position $[15,16]$. The final spatial resolution of the thermometry images was $0.72 \mathrm{~mm}$, experimentally determined by the full width half maximum of the line spread function measured using a scanning edge technique.

A ratio image was computed from each filtered image pair which was then divided by an average ratio image obtained in the gas-phase at room temperature to account for spatial non-uniformity in light collection efficiency. It should be noted that this ratio-based technique does not require a uniform beam profile. To calibrate the ratio response with temperature for this specific detection setup, a thermocouple was positioned in the measurement plane during steady operation of the jet at different exit temperatures. The thermocouple readings were compared to mean intensity ratios obtained at the same location and a quadratic fit to this calibration data was used to convert the ratio images to temperature.

\subsection{Velocimetry}

For PIV, a dual-cavity, frequency doubled diode-pumped Nd:YAG laser (Edgewave, IS-611DE) with a pulse duration of $5 \mathrm{~ns}$ and a pulse energy of $0.65 \mathrm{~mJ}$ was used. The laser was operated at $3 \mathrm{kHz}$ with a pulse separation of $50 \mu \mathrm{s}$, equally bracketing the phosphorescence excitation light pulse for quasi-simultaneous measurements. All lasers and cameras were triggered using a trigger clock and the start of the recording for the three cameras was synchronized via a custom-made manual trigger switch unit. The $532 \mathrm{~nm}$ laser beams were superimposed on the $355 \mathrm{~nm}$ beam using a dichroic mirror placed before the sheet optics. Before overlapping the beams, the width of the $355 \mathrm{~nm}$ beam was adjusted with the telescope to match the thickness of the $532 \mathrm{~nm}$ sheet $(600 \mu \mathrm{m})$. Light scattered by the particles was imaged using a third CMOS camera (Photron SA1.1, 12 bits, $1,024 \times 1,024$ pixels) and equipped with a Scheimpflug adapter, a $105 \mathrm{~mm} f / 2.8 \mathrm{Ni}$ kon lens with the $f$-stop at $f / 11$ and a $532-10 \mathrm{~nm}$ bandpass filter. The camera was operated at $6 \mathrm{kHz}$, with the laser pulses timed so that the first laser cavity is triggered at the end of the exposure of the first frame and the second laser cavity is triggered at the beginning of the exposure of the second frame, resulting in double frame acquisition at the desired $3 \mathrm{kHz}$. The camera was positioned on the opposite side of the measurement plane at a $6^{\circ}$ angle to prevent any interference between the two detection systems. Particle images were mapped to the phosphorescence images and then processed using a multi-pass cross-correlation algorithm (LaVision, $\mathrm{DaVis}$ 7.2) with an interrogation window size of $32 \times 32$ and $50 \%$ overlap.

It should be noted that the thermometry measurement could have been performed at higher frequencies without any decrease in the signal to noise ratio, as the pulse energy of the $355 \mathrm{~nm}$ laser is constant up to $10 \mathrm{kHz}$. In this experiment, the sampling rate for simultaneous measurements was limited by the PIV camera, which operated at nearly full frame readout to achieve comparable spatial resolution between the two measured fields.

\section{Results and discussion}

\subsection{Accuracy and precision of high-speed phosphor thermometry}

The use of high-speed cameras for quantitative measurements has previously been investigated using a similar 
camera of an earlier generation (Photron SA1.1) [9]. Nonlinearity was found to increase with increasing signal and to be $<5 \%$ over the maximum dynamic range of the camera (12 bits) and the pixel-to-pixel variation in gain was within $1 \%$. This was expected to have little impact in the present case, where the recorded intensity after dark image subtraction was always below 500 counts. In order to verify that the cameras used here have similar characteristics in this intensity range, a phosphor pellet was placed behind two diffusive glass screens and illuminated by the laser at $100 \mathrm{~Hz}$. At this frequency, no laser-induced heating effects were observed as the intensity ratio remained the same as in the gas-phase experiments at room temperature. As in later experiments, the cameras were allowed to thermally stabilize and images were recorded using the two-camera system at a repetition rate of $3 \mathrm{kHz}$ to exclude effects of different recording frequencies on camera performance. Different pulse energies of the laser were used to cover the range of phosphorescence signals encountered in the actual gas-phase measurements, with recorded intensities between 20 and 500 counts. Since the ratio is 0.2 at room temperature, non-linearity will be reflected by differences between ratios obtained at different illumination levels, and pixel-to-pixel variations in gain will be reflected in spatial variations when dividing two ratio images. To decrease the influence of statistical noise, an average of 100 single shots was compiled for each illumination level.

Considering a homogenously illuminated region of $600 \times 600$ pixels, the spatially averaged ratio at each illumination level differed by $<0.1 \%$, indicating negligible non-linearity in the mentioned range. The pixel-to-pixel standard deviation of the divided ratio images was $3.1 \%$. Both findings are in good agreement with the previously mentioned study [9], when propagating the uncertainties for comparison with the divided ratio image evaluation used here. The spatial variation in pixel gain was reduced to $0.6 \%$ after the images were processed, so no image correction was performed in the actual gas-phase experiments.

The camera supplier provides an intensity calibration feature to correct for differences in pixel gain and dark levels based on an acquired dark image. This was disabled for this study since it modifies the individual pixel gain after each calibration, which must be avoided for repeatability. Instead, a manual background subtraction was performed as mentioned above.

Initial measurements also indicated frame-to-frame gain fluctuations across the entire chip causing frame-to-frame variation of the overall intensity ratio. This effect was quantified using a stable tungsten light source placed behind a diffusive glass screen. Based on a homogeneously illuminated region comprised of 50 processed pixels in the centre of each intensity ratio image, the frame-to-frame standard deviation of the spatially averaged ratio was found to be $1.5 \%$. This is larger than the single shot pixel-topixel standard deviation in the same area $(0.7 \%)$, indicating these frame-to-frame fluctuations are an order of magnitude larger than the $0.01 \%$ expected when considering a statistical distribution of the sampled mean. To account for this effect in the present work, a region in the image at a known temperature was used to correct for frame-to-frame differences in the overall ratio. However, according to the manufacturer, the next generation of cameras features improved stability in the internal power supply, resulting in much smaller frame-to-frame gain fluctuations. This will decrease the measurement uncertainty caused by these fluctuations, which will make the mentioned correction unnecessary.

The single shot precision of the thermometry technique at $3 \mathrm{kHz}$ was determined from imaging data recorded in the heated jet test case at four steady jet exit temperatures. At each temperature, from 100 uncorrelated images 20,000 independent measurements were sampled from an $8.8 \mathrm{~mm} \times 8.8 \mathrm{~mm}$ region in the jet potential core. At the highest axial position in this region the jet core was $9.5 \mathrm{~mm}$ wide, ensuring no turbulent temperature fluctuations influenced the results. These statistics were used to compile the histogram shown in Fig. 3. The single shot pixel-to-pixel standard deviation was found to be $4.9 \mathrm{~K}$ $(1.7 \%), 7.8 \mathrm{~K}(2.2 \%), 9.2 \mathrm{~K}(2.2 \%)$ and $21.9 \mathrm{~K}(4.4 \%)$ at 293, 363, 423, and $500 \mathrm{~K}$, respectively. Signal statistics predict an error of $3.8 \mathrm{~K}$ at $293 \mathrm{~K}$, indicating these results are close to the noise limit. From repeated measurement sequences, the maximum deviation of the mean measured temperatures to the flow temperature indicated by a thermocouple positioned in the measurement plane was only $5 \mathrm{~K}$.

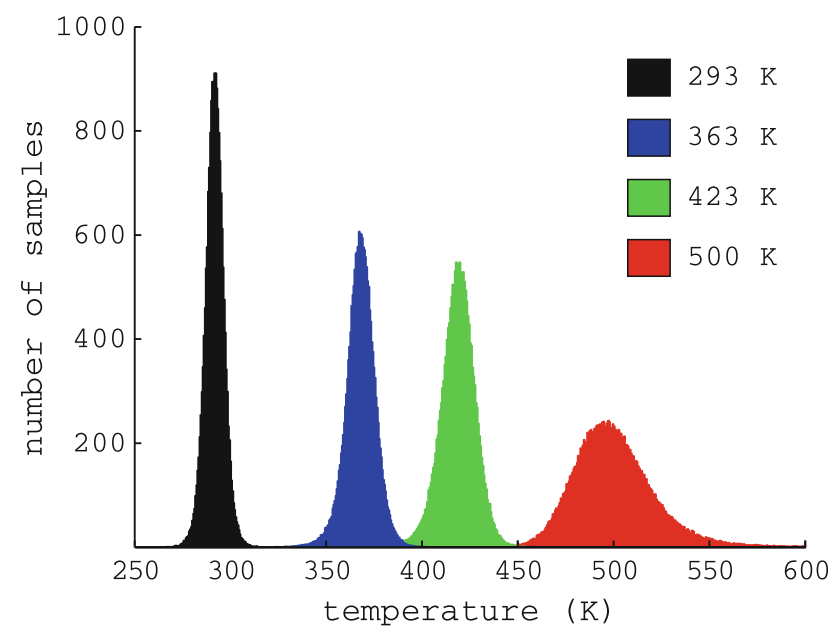

Fig. 3 Histograms of 20,000 independent measurements, recorded at $3 \mathrm{kHz}$ for each jet temperature 

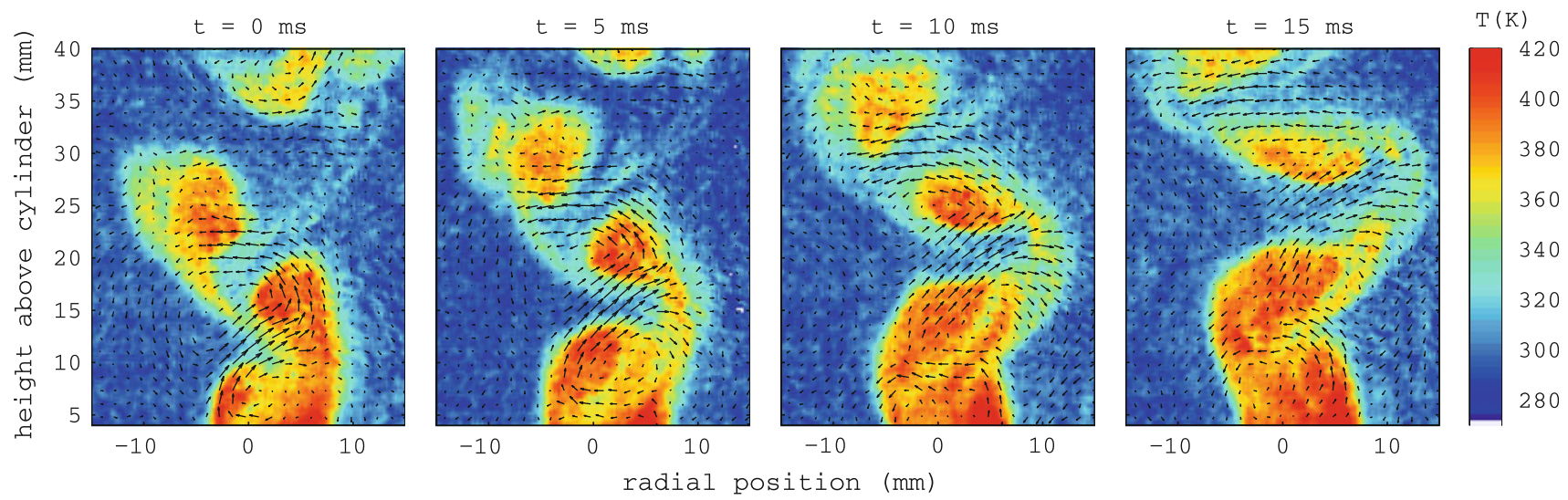

Fig. 4 Temporal evolution of the temperature and velocity fields in the wake of a heated cylinder. Every fifteenth image of the $3 \mathrm{kHz}$ recording is displayed (area is $30 \times 36 \mathrm{~mm}^{2}, 4 \mathrm{~mm}$ above the cylinder)

This level of temperature precision is comparable with that achieved in a previous study using a low speed measurement system based on interline transfer CCD cameras and low repetition rate flashlamp pumped solid-state lasers [11]. BAM:Eu saturates at fluences around $2 \mathrm{~mJ} / \mathrm{cm}^{2}$ [11], so the phosphorescence emission intensity is comparable for the two cases as saturation can also be achieved with the high-speed $355 \mathrm{~nm}$ laser. The readout noise of CMOS cameras is larger than that of the CCD cameras used in [11]. However, in this study this potential decrease in signal to noise ratio was compensated for by increasing the phosphorescence collection efficiency. The spectrally flat 50:50 beamsplitter used in the former study was replaced by a dichroic beamsplitter, almost doubling the collection efficiency, and a filter with increased transmission and a larger passband was also used which increased the light level in one channel by an additional factor of four.

\subsection{Combined measurements in a non-isothermal vortex street}

To demonstrate the utility of the combined diagnostics for the investigation of unsteady heat transfer phenomena, the time-resolved temperature and velocity fields were measured in the wake of the heated cylinder described in Sect. 2.1. Figure 4 shows four temperature and velocity fields, each separated by $5 \mathrm{~ms}$. The mean velocity field has been subtracted from the instantaneous fields to better visualize the movement of eddies, and only every fifteenth image from the original $3 \mathrm{kHz}$ recording is displayed so that a longer part of one vortex shedding cycle can be seen. Counter-rotating eddies of hot gas are alternately shed from either side of the rear stagnation point, which then cool as they are convected away from the cylinder. A video of this flow covering $50 \mathrm{~ms}$ at the full $3 \mathrm{kHz}$ recording rate is available as electronic supplementary material, allowing the visualization of two full vortex shedding cycles.

\section{Conclusion}

Simultaneous planar measurements of gas temperature and velocity have been demonstrated at sustained $\mathrm{kHz}$ repetition rates, using phosphor thermometry and a conventional PIV approach based on a single seeded tracer. Apart from a conventional high-speed PIV system, one frequency tripled laser and two non-intensified high-speed cameras are needed. The CMOS cameras were characterized for ratiobased imaging and shown to be suitable for quantitative measurements. The use of a fast, high quantum yield phosphor together with an efficient filter combination permits precise temperature measurements at $\mathrm{kHz}$ rates, despite the low pulse energy of the high-speed $355 \mathrm{~nm}$ laser. The study demonstrates the potential of this technique for time-resolved investigations of unsteady heat transfer phenomena.

Acknowledgments The authors would like to thank LaVision $\mathrm{GmbH}$ for the loan of two high-speed cameras. Financial support of the UK Engineering and Physical Sciences Research Council (EPSRC) is gratefully acknowledged.

Open Access This article is distributed under the terms of the Creative Commons Attribution License which permits any use, distribution, and reproduction in any medium, provided the original author(s) and the source are credited.

\section{References}

1. M. Löffler, S. Pfadler, F. Beyrau, A. Leipertz, F. Dinkelacker, Y. Huai, A. Sadiki, Flow. Turb. Combust. 81, 205-219 (2008)

2. S. Pfadler, J. Kerl, F. Beyrau, A. Leipertz, A. Sadiki, J. Scheuerlein, F. Dinkelacker, Proc. Combust. Inst. 32, 1723-1730 (2009)

3. B. Böhm, C. Heeger, R.L. Gordon, A. Dreizler, Flow Turbulence Combust. 86, 313-341 (2010)

4. V. Sick, Proc. Combust. Inst. 34, 3509-3530 (2013) 
5. B. Peterson, D.L. Reuss, V. Sick, Proc. Combust. Inst. 33, 3089-3096 (2011)

6. B. Böhm, C. Heeger, I. Boxx, W. Meier, A. Dreizler, Proc. Combust. Inst. 32, 1647-1654 (2009)

7. M. Cundy, P. Trunk, A. Dreizler, V. Sick, Exp. Fluids 51, 1169-1176 (2011)

8. B. Peterson, E. Baum, B. Böhm, V. Sick, A. Dreizler, Proc. Combust. Inst. 34, 3653-3660 (2013)

9. V. Weber, J. Brübach, R.L. Gordon, A. Dreizler, Appl. Phys. B. 103, 421-433 (2011)

10. A. Omrane, P. Petersson, M. Aldén, M.A. Linne, Appl. Phys. B. 92, 99-102 (2008)
11. B. Fond, C. Abram, A.L. Heyes, A.M. Kempf, F. Beyrau, Opt. Express 20, 22118-22133 (2012)

12. S.W. Allison, G.T. Gillies, Rev. Sci. Instrum. 68, 2615-2650 (1997)

13. M. Aldén, A. Omrane, M. Richter, G. Särner, Prog. Energy Combust. Sci. 37, 422-461 (2011)

14. C.H.K. Williamson, Annu. Rev. Fluid Mech. 28, 477-539 (1996)

15. A.M. Steinberg, J.F. Driscoll, S.L. Ceccio, Exp. Fluids 47, 527-547 (2009)

16. S. Pfadler, J. Kerl, F. Beyrau, A. Leipertz, A. Sadiki, J. Scheuerlein, F. Dinkelacker, Proc. Combust. Inst. 32, 1723-1730 (2009) 